\title{
Validity of a Smartphone Application for Measuring Ankle Plantar Flexion
}

\author{
Robert W. Cox, Rodrigo E. Martinez, Russell T. Baker, and Lindsay Warren
}

\begin{abstract}
Context: Range of motion is a component of a physical examination used in the diagnostic and rehabilitative processes. Following ankle injury and/or during research, it is common to measure plantar flexion with a universal goniometer. The ease and availability of digital inclinometers created as applications for smartphones have led to an increase in using this method of range of motion assessment. Smartphone applications have been validated as alternatives to inclinometer measurements in the knee; however, this application has not been validated for plantar flexion in the ankle. Objectives: The purpose of this study was (1) to assess the validity of the Clinometer Smartphone Application ${ }^{\mathrm{TM}}$ produced by Plaincode App Development for use in the ankle (ie, plantar flexion) and (2) to assess the validity of the inclinometer procedures used to measure ankle dorsiflexion for measuring ankle plantar flexion. Design: Blinded repeated measures correlational design. Setting: University-based outpatient rehabilitative clinic. Participants: A convenience sample $(\mathrm{N}=50)$ of participants $(27$ females and 23 males $)$ who reported to the clinic (mean age $=30.48$ y). Intervention: Patients were long seated on a plinth, with the knee in terminal extension. Three plantar flexion measurements were taken with a goniometer on each foot by the primary researcher. The primary researcher then conducted 3 blinded measurements with The Clinometer Smartphone Application ${ }^{\mathrm{TM}}$ following the same procedure. A second researcher, who was blinded to the goniometer measurements, recorded the inclinometer measurements. After data were collected, a Pearson's correlation was calculated to determine the validity of the clinometer app compared with goniometry. Main Outcome Measure: Degrees of motion for ankle plantar flexion. Results: Measurements produced using the Clinometer Smartphone Application ${ }^{\mathrm{TM}}$ were highly correlated for right foot $(r=.92, P<.001)$, left foot $(r=.92, P<.001)$, and combined $(r=.92, P<.001)$ with goniometer measurements using a plastic universal goniometer. Conclusion: The Clinometer Smartphone Application ${ }^{\mathrm{TM}}$ is a valid instrument for measuring plantar flexion of the ankle.
\end{abstract}

Keywords: clinical-evaluation, correlation, joint-instability, range-of-motion, foot

Range of motion (ROM) assessment is used across diagnostic and rehabilitative processes to enhance and inform both insurance reimbursement and research studies. Common ROM assessment includes aligning the fulcrum of a plastic universal goniometer with the center of the joint being measured, the stationary arm with the proximal limb, and the movement arm with the distal limb. Ankle plantar flexion is measured with the fulcrum centered over the lateral malleolus, stationary arm aligned with the fibula, and movement arm aligned with the fifth metatarsal. ${ }^{1-3}$

Inclinometry, an alternative to goniometry, is performed in a similar manner to goniometry, but the inclinometer is aligned with the distal limb to measure motion. ${ }^{2,4,5}$ The ease and availability of digital inclinometers, specifically those created as smartphone applications, have anecdotally increased the use of inclinometry for ROM assessment. Various applications have been validated as alternatives to inclinometer measurements in the knee. ${ }^{6}$ The Clinometer Smartphone Application ${ }^{\mathrm{TM}}$ produced by Plaincode App Development has been found reliable for measuring shoulder ROM, ${ }^{7}$ but it lacks validation for use in the ankle.

Inclinometry has been validated for measuring dorsiflexion in the long sitting position, with the knee in terminal extension and the inclinometer aligned with the fifth metatarsal of the foot being measured $^{4,5}$; however, this procedure, as well as the Clinometer Smartphone Application ${ }^{\mathrm{TM}}$, has not yet been validated for measuring ankle plantar flexion. The purpose of this study was 2-fold:

The authors are with the University of Idaho, Moscow, ID. Cox (utahsmartclinic@ gmail.com) is corresponding author.
(1) to assess the validity of the Clinometer Smartphone Application $^{\mathrm{TM}}$ for use in the ankle (ie, plantar flexion) and (2) to assess the validity of the inclinometer procedures used to measure ankle plantar flexion.

\section{Methods}

A blinded repeated measures correlation study was designed to determine if the Clinometer Smartphone Application ${ }^{\mathrm{TM}}$ would produce equivalent measurements to the Baseline Evaluation Instruments $^{\mathrm{TM}} 12-1000$ plastic goniometer.

The University of Idaho Institutional Review Board granted approval for the study. Written informed consent was obtained from all participants. In the case of minors, assent was received from the minor and consent was obtained from the parents/legal guardian who also observed data collection. We screened a convenience sample of individuals who reported to a musculoskeletal pain clinic with varying conditions as potential participants. Individuals were invited to participate if they could successfully maintain positioning to perform the ROM tests. As the study's purpose was to validate methods and instrumentation used to measure plantar flexion, no further inclusion or exclusion criteria were used to achieve a sample of patients. Fifty patients ( 27 females and 23 males) with a mean age of 30.48 years (range $=8-71 \mathrm{y}$ ) participated. All 50 participants accepted and completed the study in its entirety. Each of the participants completed goniometric and inclinometer assessment on each foot, providing a total sample of 100 measurements for analysis. 
Testing was conducted by asking each patient to maintain a long sitting position on a plinth. A thin bolster was placed beneath the distal tibia, allowing for terminal knee extension and the fibula to be parallel to the surface of the plinth, maintained by an assistant rater using an inclinometer, during all measurements. The goniometer was aligned in the following manner: (1) a mark was placed for the stationary arm of the goniometer at the midline proximal fibula in-line with the head of the fibula, (2) the fulcrum of the goniometer was placed at the center of the lateral aspect of the lateral malleolus, and (3) a line for the movement arm of the goniometer was placed on the midline of the lateral fifth metatarsal (Figure 1A). The patient was asked to actively plantar flex to end range while the measuring rater kept the stationary and mobile arms of the goniometer in-line with the markers. ${ }^{2-5}$ The measuring rater read and recorded the measurement to the nearest degree, repeating the procedure 3 times per limb.

After goniometric measurements were collected, the patient remained in the same position and the rater placed another mark posterior to the proximal head of the fifth metatarsal. The inclinometer procedures used were adapted from the methods established by Cosby and $\mathrm{Hertel}^{4}$ for inclinometer measurements for dorsiflexion. $^{5}$ The Clinometer Smartphone Application ${ }^{\mathrm{TM}}$ was calibrated to the surface of the plinth following the recommended procedures by the app. The phone was placed level resting on the plinth surface, and the clinician tapped the menu bar in the upper right corner, selecting calibration, and followed the prompts given by the app. After calibration, the phone was held in-line with the fifth metatarsal, with the base of the clinometer in-line with the mark at the head of the fifth metatarsal (Figure 1B). The patient plantar flexed, using the same procedure described for goniometer measurements. The clinometer was faced away from the rater, and a second assistant, who was blinded to the goniometer measurements, recorded the inclinometer measurements (Figure 1C).

Data were analyzed with the International Business Machines' SPSS Statistical Software (version 23.0; IBM, Armonk, NY). Validity was assessed by using Pearson's correlation to assess construct validity between the smartphone application and the goniometer, and the $t$ test was used to determine if there exists a significant difference between the measurement modes. Pearson's correlations were calculated to determine the strength of the relationship between the goniometer and clinometer measurements. Right $(\mathrm{N}=50)$ goniometric and clinometer measurements were compared, left $(\mathrm{N}=50)$ goniometric and clinometer measurements were compared, and all goniometer and clinometer measurements were pooled $(\mathrm{N}=100)$ and compared. To assess the validity of the inclinometer measurement procedures, a $t$ test was used to analyze the entire sample to determine if measurement differed between the goniometer and clinometer values. Statistical significance was set at $P \leq .05$, and the confidence interval was set at $95 \% .^{8} \mathrm{~A}$ value above .8 is considered a high correlation, or low likelihood of coincidental correlation, for validity assessment. ${ }^{8-10}$

\section{Results}

All assumptions were assessed and met for a Pearson's correlation and parametric statistics. The mean goniometric measurement of plantar flexion was 62.79 ( 7.90) across all ankles; the mean clinometer measurement was 62.96 (7.85) (Table 1). There was a high correlation between the 2 measurement devices on the right ankle $(r(50)=.92, P<.001)$, the left ankle $(r(50)=.92, P<.001)$, and the pooled samples $(r(100)=.92, P<.001)$ (Table 1$)$. The $t$-test results indicated that there were no significant difference across the measuring devices, with a mean difference of -0.18 (95\% confidence interval, -0.799 to 0.439$), t(100)=-.577, P=.57$ (Table 2).

\section{Discussion}

In a technologically advancing world, smartphones are commonplace in many settings. The smartphone application is a convenient way for clinicians to quickly measure ROM. Applications can be downloaded on any smartphone making it a practical tool, assuming the clinician has access to a smartphone, an understanding of proper inclinometer procedures, and the applications are reliable and valid. Previous findings have indicated that the Clinometer Smartphone Application ${ }^{\mathrm{TM}}$ is a reliable inclinometer device for assessing shoulder ROM. ${ }^{7}$ Furthermore, inclinometry has been established as a valid alternative to goniometry for measuring dorsiflexion, ${ }^{4,5}$ but this has not been established for plantar flexion.

The high correlation values $(r=.919, .922 \text {, and } .923)^{10}$ found between the goniometer and clinometer measurements indicate that the application is a valid tool for measuring ankle plantar flexion (Table 1). The goniometric plantar flexion measurements were not significantly different to the digital inclinometer measurements

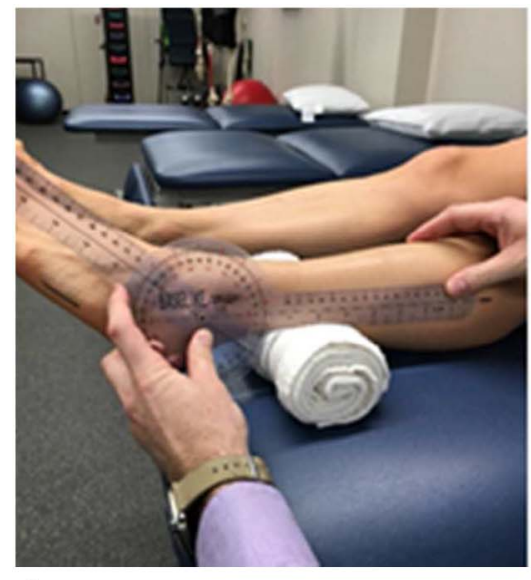

A

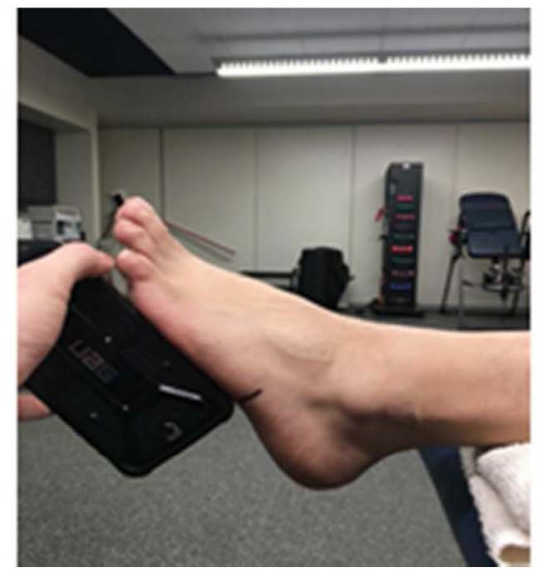

B

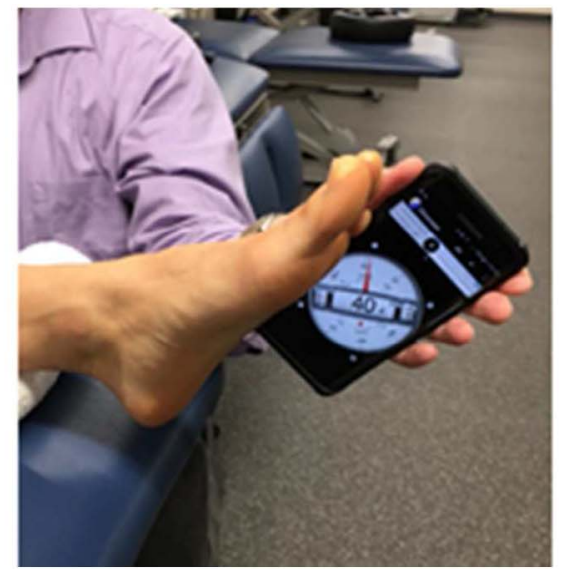

C

Figure 1 - (A) Goniometry procedure, clinician view; (B) clinometer procedure, clinician view; and (C) second observer view, blinding the clinician. 
Table 1 Descriptive Summary of ROM Measurements and Pearson's Correlation Results (95\% Confidence Interval)

\begin{tabular}{llccccccc}
\hline Ankle & & Mean & Median & Range & N & Significance & $\boldsymbol{R}^{\mathbf{2}}$ & Pearson's correlation \\
\hline Right & Goniometer & $63.33^{\circ}\left(7.92^{\circ}\right)$ & $62.33^{\circ}$ & $45.67^{\circ}-78.33^{\circ}$ & 50 & $P<.001$ & .845 & $r=.919$ \\
& Clinometer & $63.43^{\circ}\left(7.97^{\circ}\right)$ & $62.84^{\circ}$ & $44.33^{\circ}-80.67^{\circ}$ & 50 & & \\
\multirow{2}{*}{ Left } & Goniometer & $62.24^{\circ}\left(7.92^{\circ}\right)$ & $62.17^{\circ}$ & $44.33^{\circ}-78.33^{\circ}$ & 50 & $P<.001$ & .852 & $r=.923$ \\
& Clinometer & $62.51^{\circ}\left(7.77^{\circ}\right)$ & $62.67^{\circ}$ & $39.67^{\circ}-77.67^{\circ}$ & 50 & & \\
Composite & Goniometer & $62.79^{\circ}\left(7.9^{\circ}\right)$ & $62.25^{\circ}$ & $44.67^{\circ}-78.33^{\circ}$ & 100 & $P<.001$ & .85 & $r=.922$ \\
& Clinometer & $62.97^{\circ}\left(7.85^{\circ}\right)$ & $62.76^{\circ}$ & $39.67^{\circ}-80.67^{\circ}$ & 100 & & & \\
\hline
\end{tabular}

Abbreviation: ROM, range of motion.

Table 2 t-Test Results

\begin{tabular}{cccc}
\hline & $\mathbf{N}$ & Mean difference & Significance \\
\hline Goniometer-clinometer & 100 & -0.18 & $P=.57$ \\
\hline
\end{tabular}

( $\mathrm{n}=100$, mean difference $=-0.18, P=.57$; Table 2 ). The results support the use of an inclinometer as a valid alternative to a goniometer for assessing plantar flexion. Based on our findings and those previous, ${ }^{4,6,7}$ the Clinometer Smartphone Application ${ }^{\mathrm{TM}}$ can be used as a valid alternative for measuring dorsiflexion and plantar flexion of the ankle.

As with any research, there were limitations to the study. Reliability for the Clinometer Smartphone Application ${ }^{\text {TM }}$ was not established prior to this study and cannot be assumed for plantar flexion measurements. As with any measurement, there is a learning curve, and the more often a clinician can practice a skill, the better at it they will become. We recommended that clinicians establish their own reliability prior to using an inclinometer or goniometer for research or assessment of patient progress. All measurements were performed with active plantar flexion, which may or may not translate to passive ROM measurement. The measurements were made with plantar flexion only, and the study was not designed to validate inversion, eversion, or any other measurement. Most of the participants had physical conditions for which they were being actively treated at the time of study participation. Types of injuries were not recorded, and inclusion was solely based on the patient's ability to assume the position for measurements and a willingness to participate. Other demographic information such as body mass index, activity level, health condition, and weight were not assessed as part of this study. Finally, the Clinometer Smartphone Application ${ }^{\mathrm{TM}}$ is limited to the use of smartphones, and we did not assess any of the limitations related to their use (eg, screen visibility in daylight).

The Clinometer Smartphone Application ${ }^{\mathrm{TM}}$ produced by Plaincode App Development is a valid instrument for measuring ankle plantar flexion compared with a universal plastic goniometer. The application may be a valid alternative to other devices used to measure ankle ROM during research, demonstrating patient progress, and/or for any other purposes deemed appropriate. Further research is required to validate the use of this smartphone application across other joints and in comparison to other methods of ROM assessment. Although other methods and instrumentation would need to be validated in future studies, the availability and utility of smartphone applications may become valuable tools for clinicians and researchers as new technologies continue to develop.

\section{References}

1. Elveru RA, Rothstein JM, Lamb RL. Goniometric reliability in a clinical setting. Phys Ther. 1988;68(5):672-677. PubMed ID: 3362980 doi:10.1093/ptj/68.5.672

2. Norkin CC, White DJ. Measurement of Joint Motion: A Guide to Goniometry. 4th ed. Philadelphia, PA: F. A. Davis Company; 2009.

3. Starkey C, Brown SD. Orthopedic and Athletic Injury Examination Handbook. 3rd ed. Philadelphia, PA: F. A. Davis Company; 2015.

4. Cosby NL, Hertel J. Relationships between measures of posterior talar glide and ankle dorsiflexion range of motion. Athl Train Sport Health Care. 2011;3(2):76-85. doi:10.3928/19425864-20100930-02

5. Denegar CR, Hertel J, Fonseca J. The effect of lateral ankle sprain on dorsiflexion range of motion, posterior glide, and joint laxity. J Ortho Sport Phys Ther. 2002;32(4):166-173. doi:10.2519/jospt.2002.32. 4.166

6. Mourcou Q, Fleury A, Diot B, Franco C, Vuillerme N. Mobile phone-based joint angle measurement for functional assessment and rehabilitation of proprioception. BioMed Research Intern. 2015; 2015:328142. doi:10.1155/2015/328142

7. Shin SH, Ro DH, Lee OS, Oh JH, Kim SH. Within-day reliability of shoulder range of motion measurement with a smartphone. Man Ther. 2012;17:298-304. PubMed ID: 22421186 doi:10.1016/j.math.2012. 02.010

8. Jones A, Sealey R, Crowe M, Gordon S. Concurrent validity and reliability of the Simple Goniometer iPhone app compared with the Universal Goniometer. Physiother Theory Pract. 2014;30:512-516. doi:10.3109/09593985.2014.900835

9. Vincent WJ, Weir JP. Statistics in Kinesiology. 4th ed. Champaign, IL: Human Kinetics; 2012.

10. Leech NL, Barrett KC, Morgan GA. IBM SPSS for Intermediate Statistics-Use and Interpretation. 5th ed. New York, NY: Routledge; 2015. 